\title{
GMR
}

\section{Histone H3K9 acetylation influences growth characteristics of goat adipose-derived stem cells in vitro}

\author{
X. Wang*, F.X. Zhang*, Z.M. Wang, Q. Wang, H.F. Wang, Y. Ren, \\ D.P. Tai, H. Liang and D.J. Liu
}

Key Laboratory of Mammalian Reproductive Biology and Biotechnology Ministry of Education, Inner Mongolia University, Hohhot, China

*These authors contributed equally to this study.

Corresponding author: D.J. Liu

E-mail: nmliudongjun@sina.com

Genet. Mol. Res. 15 (4): gmr15048954

Received July 7, 2016

Accepted September 14, 2016

Published November 3, 2016

DOI http://dx.doi.org/10.4238/gmr15048954

Copyright $(C) 2016$ The Authors. This is an open-access article distributed under the terms of the Creative Commons Attribution ShareAlike (CC BY-SA) 4.0 License.

ABSTRACT. Adipose-derived stem cells (ADSCs) show nearly
unlimited potential in medical and animal science. Currently,
understanding of the biological mechanisms regulating ADSC growth
in vitro remains very limited. Histone acetylation, an epigenetic
modification, plays a key role in maintaining stem cell properties. To
further study its effect on ADSC growth characteristics in vitro, we
treated goat ADSCs with the histone deacetylase inhibitors trichostatin
A (TSA) and vorinostat (SAHA). This inhibited SIRT1 expression and
increased histone H3K9 acetylation, leading to decreased cell viability,
cell cycle arrest, and apoptosis. Quantitative real-time polymerase chain
reaction revealed that H3K9 hyperacetylation stimulated transcription
of $N A N O G, O C T 4, S O X 2$, and TERT, but inhibited that of PCNA, P53,
and $B A X$. Western blotting indicated that TSA and SAHA increased

Genetics and Molecular Research 15 (4): gmr15048954 
protein expression of NANOG, reduced that of SOX2, TERT, PCNA, $\mathrm{P} 53$, and BAX, and did not change that of OCT4. These findings provide new experimental evidence contributing to our understanding of the mechanisms underlying ADSC growth characteristics in vitro.

Key words: Adipose-derived stem cell; Histone acetylation; Cell proliferation; Cell apoptosis; Stem cell multipotency

\section{INTRODUCTION}

Recently, adult stem cells (ASCs) have emerged as a novel cell resource, replacing embryonic (ESCs) and umbilical cord blood stem cells owing to the relative ease with which they can be obtained and the simpler ethical considerations involved (Collas, 2010). Compared with other ASCs, those derived from adipose tissue are easy to isolate and culture, and grow rapidly. In addition, they are capable of differentiating into various cell types, including those of osteogenic, chondrogenic, adipogenic, neurogenic, hepatogenic, and endothelial lineages, depending on the stimuli and growth factors provided in vitro (Teven et al., 2011; Ahmadi et al., 2012; Culmes et al., 2013; Ge et al., 2014; Kwon et al., 2015). Therefore, adipose-derived stem cells (ADSCs) have a wide range of potential applications in tissue engineering.

Epigenetic alterations (DNA methylation and histone modifications) are heritable changes that regulate gene silencing and expression, control cellular differentiation and development, and maintain phenotypes of multicellular organisms by mechanisms other than DNA sequence changes (Dudakovic et al., 2015). Epigenetic patterns are influenced by many in vitro conditions, including passage number, serum concentration, cell density, and culture material and reagents (Long et al., 2014). Numerous studies have shown that nuclear transplantation using donor cells with reprogramming abnormalities leads to early developmental defects in the cloned embryo. Moreover, successful reprogramming of donor cells in the ooplasm depends on the epigenetic information that they carry (Lagutina et al., 2013; Ogura et al., 2013). Thus, choosing suitable donor cells can improve the efficiency of mammalian cloning. ADSCs have previously been used as donor cells for nuclear transplantation resulting in the successful production of transgenic animals (Oh et al., 2011). Therefore, such cells may be used to enhance the effectiveness of cloning and transgenic animal production.

With the increasing application of ADSCs in human medicine and animal science, researchers have become interested in studying the mechanism underlying their in vitro growth characteristics (Latella et al., 2012; Wankhade et al., 2016). In recent years, many studies have shown that ASCs are affected by various epigenetic modifications, which play key roles in regulating gene silencing and expression in mammalian cells and maintaining the capacity for self-renewal and multi-directional differentiation (Yannarelli et al., 2013; Rinaldi and Benitah, 2015). Histone modification is a highly dynamic process with diverse biological effects. In particular, the function of histone acetylation as a marker of gene expression in cell fate has become a major research focus (Zhang et al., 2012).

In this study, we investigated the epigenetic mechanism determining the growth characteristics of ADSCs from cashmere goats. We employed two commonly used histone deacetylase inhibitors (HDACIs), trichostatin A (TSA) and vorinostat (SAHA), to modify the effect of HDAC proteins (HDAC1, HDAC2, and SIRT1) in Arbas cashmere goat ADSCs (gADSCs). We analyzed cell viability, cell cycle phase distribution, and apoptosis after TSA

Genetics and Molecular Research 15 (4): gmr15048954 
and SAHA treatment, determined the level of $\mathrm{H} 3 \mathrm{~K} 9$ acetylation, and measured the transcription and translation of genes related to multipotency, proliferation, and apoptosis. Our results contribute to an understanding of the mechanisms underlying the in vitro growth characteristics of ADSCs, and provide an experimental basis for applications such as mammalian cloning and transgenic animal production.

\section{MATERIAL AND METHODS}

\section{Materials}

Primary Arbas cashmere goat ADSCs were isolated and preserved in liquid nitrogen by our team (Ren et al., 2012). The fifth generation of these cells was passaged in the present study. Dulbecco's modified Eagle's medium (DMEM) and phosphate-buffered saline (PBS) were obtained from HyClone (Logan, UT, USA). Fetal bovine serum (FBS), trypsin/ethylenediaminetetraacetic acid, and 2-mercaptoethanol were purchased from Gibco (Grand Island, NY, USA). TSA and SAHA were obtained from Selleckchem (Houston, TX, USA). Anti-alpha tubulin, anti-histone H3 (acetyl K9), anti-HDAC1, anti-HDAC6, and anti-SIRT1 antibodies, and fluorescein isothiocyanate (FITC)- and horseradish peroxidase (HRP)-labeled goat anti-rabbit polyclonal antibodies were purchased from Abcam (Cambridge, UK). Anti-NANOG, anti-OCT4, anti-SOX2, anti-TERT, anti-PCNA, anti-P53, and anti-BAX antibodies were obtained from Proteintech (Chicago, IL, USA). RNAiso Plus, PrimeScript RT Master Mix, and a PrimeScript RT reagent Kit with gDNA Eraser were obtained from TaKaRa (Tokyo, Japan). Mammalian protein extraction reagent, sodium dodecyl sulfate-polyacrylamide gel electrophoresis (SDS-PAGE) electrophoretic solution, and transfer membrane solution were purchased from Cwbiotech (Beijing, China). Tissue culture dishes were purchased from Corning (Corning, NY, USA).

\section{gADSC growth curve}

gADSCs were seeded on 24-well culture plates at a concentration of $1 \times 10^{3}$ cells $/ \mathrm{cm}^{2}$ and cultured in DMEM supplemented with $20 \%$ FBS. All cultures were incubated at $37^{\circ} \mathrm{C}$ in a humidified atmosphere containing $5 \% \mathrm{CO}_{2}$, and the medium was changed twice per week. Cell morphology was observed under an inverted microscope (Zeiss, Observer.A1). Cells were counted every day following attachment for $24 \mathrm{~h}$. After 7 days, a cell growth curve was drawn.

\section{Thiazolyl blue tetrazolium bromide (MTT) assay}

Briefly, gADSCs in the logarithmic growth phase were seeded on 96-well plates and incubated in $100 \mu \mathrm{L}$ DMEM/F12 supplemented with $20 \%$ FBS. After $24 \mathrm{~h}, 500 \mathrm{nM}$ TSA or $16 \mu \mathrm{M}$ SAHA was added to the medium, and cells were incubated for a further 24,48 , or $72 \mathrm{~h}$. Subsequently, cells were exposed to $10 \mu \mathrm{L}$ MTT solution $(5 \mathrm{mg} / \mathrm{mL})$ for $4 \mathrm{~h}$ at $37^{\circ} \mathrm{C}$. DMSO $(100 \mu \mathrm{L})$ was then added and incubated with cells for $5 \mathrm{~min}$ to dissolve the formazan crystals. Absorbance at $490 \mathrm{~nm}$ was measured with a microplate reader and used to calculate cell viability.

\section{Cell cycle and apoptosis measurements}

Cells with or without $500 \mathrm{nM}$ TSA or $16 \mu \mathrm{M}$ SAHA treatment were collected after 24,

Genetics and Molecular Research 15 (4): gmr15048954 
48, and $72 \mathrm{~h}$. Untreated cells were used as the control group. Cells were stained with propidium iodide (PI) and annexin V-FITC/PI. Flow cytometry was then used to analyze apoptosis rate and the distribution of cells in each cycle phase.

\section{Immunocytochemistry}

Cells were fixed with $4 \%$ paraformaldehyde, and after being washed with PBS, were permeabilized with $0.5 \%$ Triton $\mathrm{X}-100$ at $25^{\circ} \mathrm{C}$ for $1 \mathrm{~h}$ and blocked with $10 \%$ goat serum, also at room temperature for $1 \mathrm{~h}$. Cells were subsequently incubated overnight at $4^{\circ} \mathrm{C}$ with primary antibodies (diluted 1:300), which were replaced by PBS in the negative control group. The cells were then incubated for $1 \mathrm{~h}$ at room temperature with FITC-conjugated secondary antibodies (diluted 1:300), before being stained for 2 min with 4',6-diamidino-2-phenylindole (diluted 1:1000) and observed under a confocal laser scanning microscope to determine target protein expression.

\section{Total RNA extraction and reverse transcription}

Cells were collected in microtubes. RNAiso Plus was added, and after being vortexed, the tubes were left for $5 \mathrm{~min}$ at room temperature. Chloroform was then added, and the samples were vortexed and centrifuged at $12,000 \mathrm{rpm}$ for $15 \mathrm{~min}$ at $4^{\circ} \mathrm{C}$. The supernatants were transferred to new clear microtubes, to which an equal volume of isopropanol was added. Samples were then mixed and centrifuged before discarding the supernatants. The precipitates were washed with $75 \%$ ethanol, dried briefly at room temperature, and resuspended in RNase-free water. The concentration and purity of total RNA extracts were determined, and each sample was required to have a 260/280 nm optical density ratio between 1.8 and 2.0. Complementary DNA (cDNA) was prepared from total RNA using a PrimeScript RT reagent Kit with gDNA Eraser following the manufacturer protocol, before being stored at $-20^{\circ} \mathrm{C}$.

\section{Quantitative real-time polymerase chain reaction (qPCR)}

mRNA expression was measured on an ABI 7500 Real-Time PCR System (Applied Biosystems, Foster City, CA, USA), with actin as the reference gene. Following the manufacturer protocol, each reaction comprised $12.5 \mu \mathrm{L}$ SYBR Premix Ex Taq II, $1.0 \mu \mathrm{L}$ each forward and reverse primer, $2.0 \mu \mathrm{L}$ cDNA template, and $8.5 \mu \mathrm{L}$ RNase-free water. Cycling conditions consisted of an initial denaturation at $95^{\circ} \mathrm{C}$ for $30 \mathrm{~s}$, followed by 50 cycles of denaturation at $95^{\circ} \mathrm{C}$ for $5 \mathrm{~s}$ and annealing at $60^{\circ} \mathrm{C}$ for $31 \mathrm{~s}$. Fluorescence was measured after each cycle.

\section{Western blot}

Cells were harvested by trypsinization and lysed with cell lysis buffer containing protease inhibitor. Protein concentration was determined by BCA assay. Proteins were separated by SDS-PAGE ( $90 \mathrm{~V}$ for $30 \mathrm{~min}$, then $120 \mathrm{~V}$ for $90 \mathrm{~min}$ ) and transferred to nitrocellulose membranes. After being blocked with 5\% nonfat dry milk for $2 \mathrm{~h}$ at room temperature, membranes were incubated with primary antibodies (diluted 1:1000) overnight at $4{ }^{\circ} \mathrm{C}$. Thereafter, membranes were rinsed with Tris-buffered saline-Tween 20 three times by

Genetics and Molecular Research 15 (4): gmr15048954 
shaking, and incubated with HRP-conjugated secondary antibodies for $1 \mathrm{~h}$ at room temperature. Proteins were visualized using an ECL Prime detection kit (GE Healthcare, Little Chalfont, UK).

\section{Statistical analysis}

The SPSS software (SPSS Inc., Chicago, IL, USA) was used for statistical analysis of experimental data, and $\mathrm{P}<0.05$ was considered to represent a significant difference. Each experiment was carried out independently at least three times.

\section{RESULTS}

\section{Growth curve and MTT assay analysis of cell viability}

The 7-day growth curve conformed to the classic sigmoid pattern of cell proliferation, consisting of four phases: lag (0-48 h), logarithmic (48-96 h), stationary (96-144 h), and decline (144-168 h; Figure 1A).

After exposure to medium containing $500 \mathrm{nmol} / \mathrm{L}$ TSA or $16 \mathrm{mmol} / \mathrm{L}$ SAHA for 24 , 48, or $72 \mathrm{~h}$, cell viability was evaluated by MTT assay (Figure 1B and 1C). OD 490-nm ratio meant cell viability. Cell viability measurements were $0.734 \pm 0.015$ and $0.678 \pm 0.074$ at $24 \mathrm{~h}$, $0.497 \pm 0.066$ and $0.588 \pm 0.114$ at $48 \mathrm{~h}$, and $0.467 \pm 0.064$ and $0.411 \pm 0.080$ at $72 \mathrm{~h}$ following TSA and SAHA treatment, respectively. Thus, cell viability was affected by TSA and SAHA in a time-dependent manner, being highest at $24 \mathrm{~h}$, and subsequently decreasing.

A

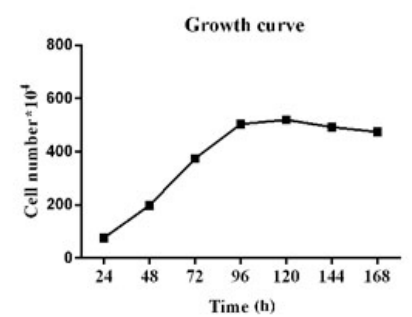

B

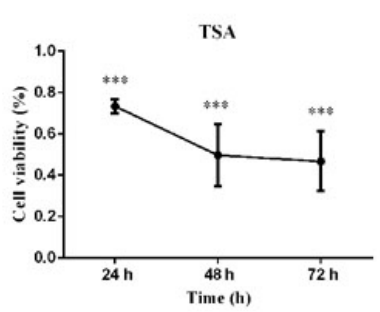

C

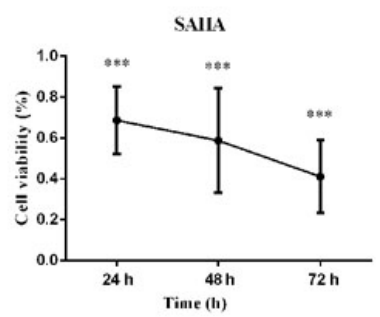

Figure 1. Cell growth curve and viability analysis. A. Growth curve of Arbas cashmere goat adipose-derived stem cells. B. Cell viability after treatment with $500 \mathrm{nmol}$ trichostatin A (TSA). C. Cell viability after treatment with 16 mmol vorinostat (SAHA). Each experiment was carried out independently at least three times. $* * * \mathrm{P}<0.01$.

\section{Flow cytometric analysis of cell cycle phase distribution and apoptosis rate}

We used flow cytometry to analyze apoptosis rates and the distribution of gADSCs in each cell cycle phase with or without HDACI treatment for 24, 48, and $72 \mathrm{~h}$.

PI staining is an indicator of cell DNA content, and can be used to analyze cell cycle phase distribution (Figure 2A). Compared with untreated cells, exposure to TSA or SAHA for $24 \mathrm{~h}$ significantly increased the percentage of cells in $\mathrm{G} 0 / \mathrm{G} 1$ by $16.415 \pm 1.1$ and 17.695 $\pm 0.53 \%$, decreased that of those in S phase by $1.135 \pm 1.1$ and $4.615 \pm 0.53 \%$, and reduced that of those in $\mathrm{M}$ phase by $13.35 \pm 1.1$ and $11.075 \pm 0.53 \%$, respectively. After treatment with TSA and SAHA for $48 \mathrm{~h}$, the proportion of cells in G0/G1 fell by $6.28 \pm 0.01$ and $2.75 \pm$ 
$0.03 \%$, that of those in S phase increased by $0.82 \pm 0.01$ and $0.26 \pm 0.03 \%$, and that of those in $\mathrm{M}$ phase grew by $1.78 \pm 0.01$ and $2.56 \pm 0.03 \%$, respectively, but these changes were not significant. Owing to serious cell damage, fewer cells were detected after $72 \mathrm{~h}$.

The effects of TSA and SAHA on gADSC apoptosis are shown in Figure 2B. Compared with the control group, TSA treatment for $24 \mathrm{~h}$ significantly increased the percentage of cells in early apoptosis by $28.78 \pm 2.45 \%$, and raised the apoptosis ratio by $4.81 \pm 2.45 \%$. SAHA also significantly elevated the percentage of cells in early apoptosis, by $16.23 \pm 3.53 \%$, but decreased the apoptosis ratio by $4.63 \pm 3.53 \%$. After $48 \mathrm{~h}$ of TSA and SAHA treatment, the proportion of early apoptotic cells increased by TSA and decreased by SAHA, and that of apoptotic cells increased, but the latter was not significant. The percentage of cells undergoing apoptosis had increased somewhat after $72 \mathrm{~h}$, showing that prolonged treatment promoted gradual progression from the early to the late stage of apoptosis.
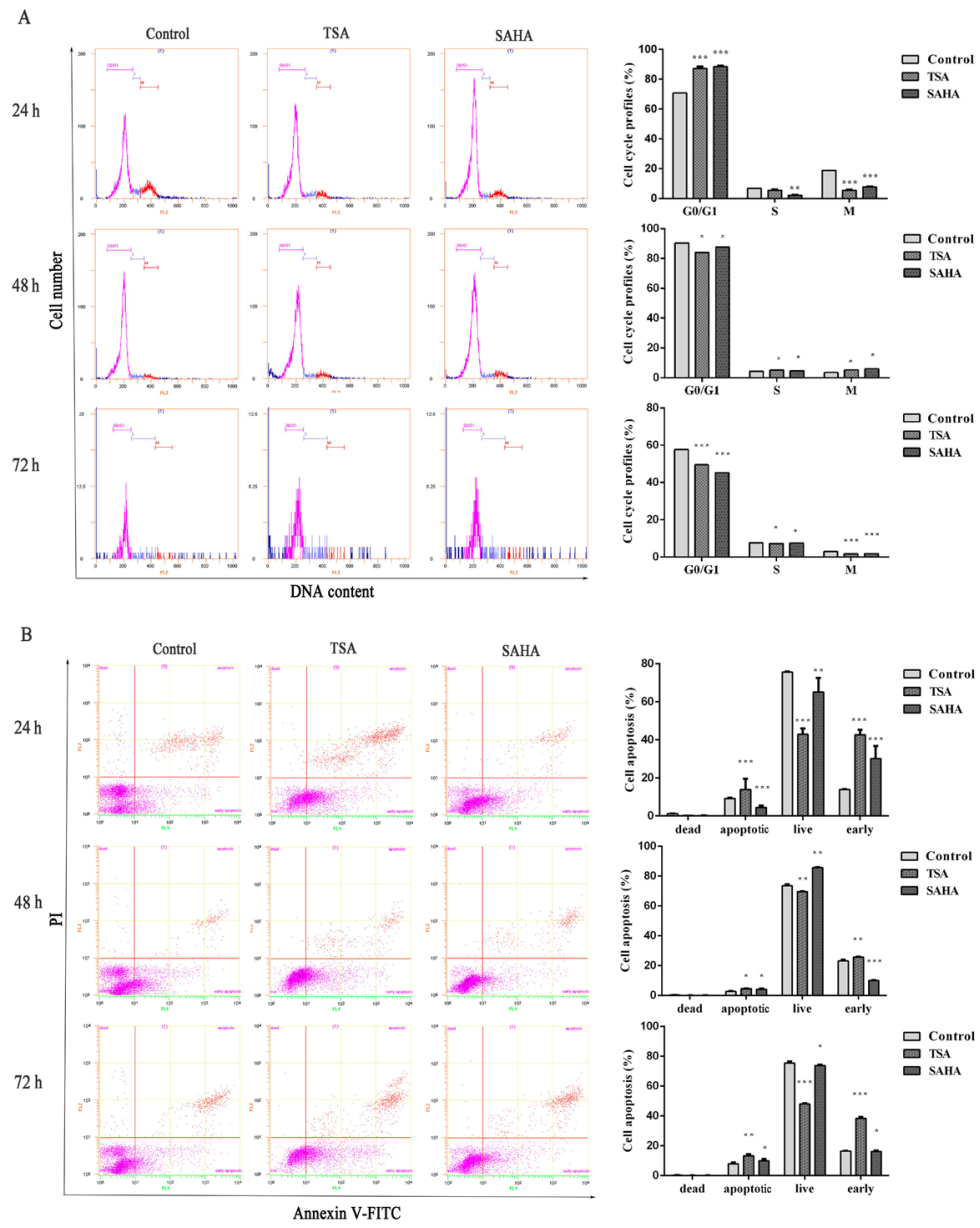

Figure 2. Cell cycle phase distribution and apoptosis analysis. A. Flow cytometric analysis of cell cycle phase distribution after treatment for 24,48 , and $72 \mathrm{~h}$. B. Flow cytometric analysis of cell apoptosis after treatment for 24, 48, and $72 \mathrm{~h}$. Each experiment was carried out independently at least three times. TSA = trichostatin A, SAHA $=$ vorinostat, $\mathrm{PI}=$ propidium iodide, $\mathrm{FITC}=$ fluorescein isothiocyanate. $* \mathrm{P}>0.05 ; * * 0.01<\mathrm{P}<0.05 ; * * * \mathrm{P}<0.01$. 


\section{Detecting presence of acetylated $\mathrm{H3K9}$ (acH3K9)}

Compared with control cells cultured in complete medium, immunocytochemical analysis of gADSCs treated with TSA or SAHA for $24 \mathrm{~h}$ revealed enhanced acH3K9-associated fluorescence (Figure 3A). Western blot analysis of untreated and treated gADSCs showed that acH3K9 levels were significantly higher in the latter, with TSA demonstrating a larger effect than SAHA in this respect (Figure 3B).
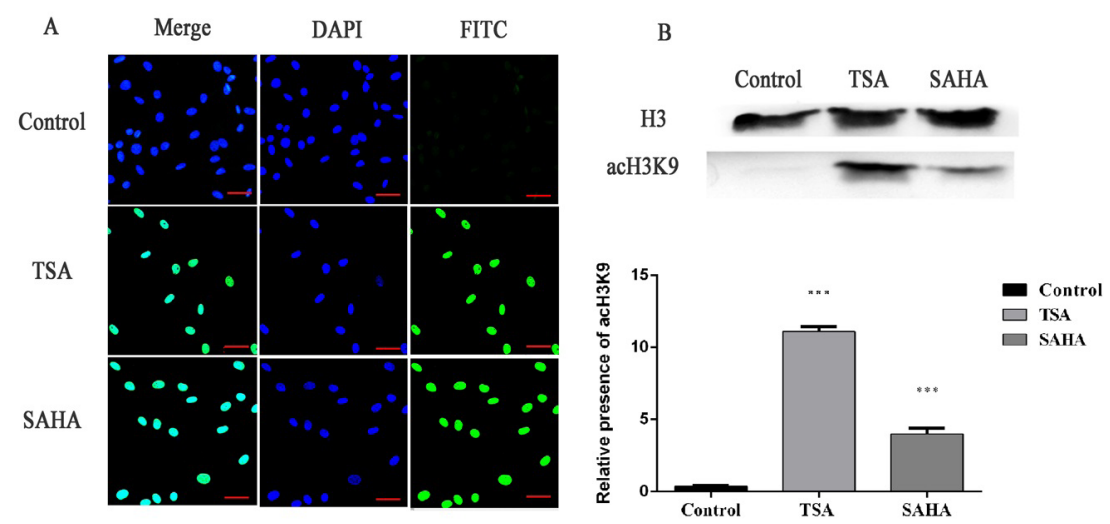

Figure 3. H3K9 acetylation analysis. A. H3K9 acetylation detected by immunocytochemistry. Bar $=50 \mathrm{~mm}$. B. H3K9 acetylation detected by western blot $(\mathrm{P}<0.01)$. TSA $=$ trichostatin $\mathrm{A}, \mathrm{SAHA}=$ vorinostat, DAPI $=$ 4',6-diamidino-2-phenylindole, FITC = fluorescein isothiocyanate, acH3K9 = acetylated H3K9. ***P $<0.01$.

\section{qPCR and western blot detection of HDAC gene and protein expression levels}

We analyzed the mRNA and protein expression levels of three HDAC genes (HDAC1, $H D A C 6$, and SIRT1) in gADSCs after TSA and SAHA treatment for $24 \mathrm{~h}$ (Figure 4). Compared with the control group, qPCR analysis revealed that TSA resulted in increased transcription of all three genes, by $2.66 \pm 0.04,4.02 \pm 0.4$, and $2.85 \pm 0.1$-fold, respectively, whereas SAHA increased $H D A C 1$ and $H D A C 6$ mRNA levels but decreased that of SIRT1, to $3.77 \pm 0.06,2.45$ \pm 0.09 , and $0.82 \pm 0.04$, respectively (Figure 4A). In addition, HDAC1, HDAC6, and SIRT1 protein expression levels relative to LAMIN B were $40.58 \pm 0.85,3.51 \pm 0.51$, and $42.15 \pm$ 0.19 in the control group, $43.14 \pm 1.13,12.0 \pm 0.13$, and $19.3 \pm 0.06$ in the TSA group, and $37.61 \pm 1.65,6.33 \pm 0.15$, and $6.27 \pm 0.06$ in the SAHA group, respectively. Western blotting indicated that TSA significantly promoted expression of HDAC1 and HDAC6 proteins, but inhibited that of SIRT1. SAHA treatment increased HDAC6 protein levels but decreased those of HDAC1 and SIRT1 (Figure 4B). Although H3K9 hyperacetylation promoted transcription of HDAC1, HDAC6, and SIRT1, TSA and SAHA inhibited translation of SIRT1.

\section{Immunocytochemistry, qPCR, and western blot detection of pluripotency, proliferation, and apoptosis-related gene expression}

Immunocytochemistry, $\mathrm{qPCR}$, and western blotting were used to detect the transcription and translation of multipotency (NANOG, OCT4, and SOX2), proliferation (TERT and PCNA), and apoptosis (P53 and BAX)-related genes after TSA and SAHA treatment for 


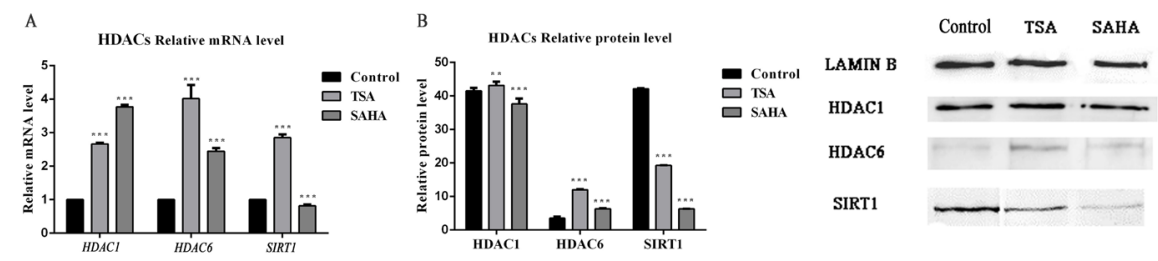

Figure 4. Relative histone deacetylase (HDAC) mRNA levels and relative HDAC protein levels. A. Quantitative real-time polymerase chain reaction was used to detect HDAC gene transcription levels in untreated and treated Arbas cashmere goat adipose-derived stem cells (gADSCs). B. Western blot was employed to measure HDAC protein levels in untreated and treated gADSCs. TSA $=$ trichostatin A, SAHA $=$ vorinostat. $* * 0.01<\mathrm{P}<0.05 ; * * * \mathrm{P}<0.01$.

$24 \mathrm{~h}$. Compared with control cells, the intensity of fluorescence associated with NANOG and TERT after treatment was weak, and OCT4 was not always expressed; however, expression of SOX2, PCNA, P53, and BAX was unchanged (Figure 5A).

mRNA expression was quantitatively analyzed by comparison with non-treated cells and normalization to the housekeeping gene $\beta$-actin. mRNA levels of NANOG, OCT4, SOX2, and TERT were significantly increased in treated cells, whereas those of $P 53$ and $B A X$ were decreased, and PCNA transcription was completely inhibited (Figure 5B).

Compared to untreated cells, those administered TSA or SAHA showed decreased expression of TERT, PCNA, and P53 proteins, and significantly increased and diminished NANOG and SOX2 levels, respectively. However, these HDACIs had no effect on OCT4 and BAX expression (Figure 5C).
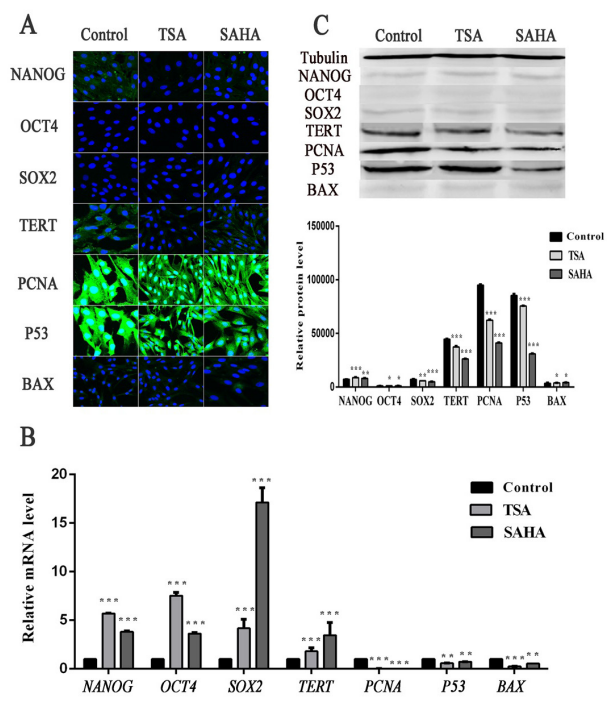

Figure 5. Expression of multipotency ( $N A N O G, O C T 4$, and $S O X 2)$, proliferation (TERT and PCNA), and apoptosis (P53 and BAX)-related genes. A. Immunocytochemistry was used to analyze NANOG, OCT4, SOX2, TERT, PCNA, $\mathrm{P} 53$, and BAX expression $(\mathrm{Bar}=50 \mathrm{~mm})$. B. Quantitative real-time polymerase chain reaction was carried out to analyze transcription levels of NANOG, OCT4, SOX2, TERT, PCNA, P53, and BAX in untreated and treated Arbas cashmere goat adipose-derived stem cells. C. Western blotting was applied to determine the relative expression levels of NANOG, OCT4, SOX2, TERT, PCNA, P53, and BAX. TSA = trichostatin A, SAHA = vorinostat. ${ }^{*} \mathrm{P}>$ $0.05 ; * * 0.01<\mathrm{P}<0.05 ; * * * \mathrm{P}<0.01$.

Genetics and Molecular Research 15 (4): gmr15048954 


\section{DISCUSSION}

The unique attributes of ADSCs in many research applications have been demonstrated in recent years. For example, using nude mice, Rodriguez et al. (2015) injected human ADSCs into a 12-mm wound, finding that these cells accelerate blood flow and promote skin healing. Lee et al. (2011) reported that human ADSCs secreting various bone cell activating factors, including hepatocyte growth factor and extracellular matrix proteins, can promote the generation of cartilage cells, increase their number in bone tissue, and prevent osteoporosis. Moreover, Leu et al. (2010) established a rat model of acute ischemic stroke, using which they demonstrated that injection of ADSCs can reduce the cerebral infarction area and improve sensorimotor function. In addition, the use of ADSCs as donor cells for nuclear transfer cloning enhances embryo development and improves blastocyst formation rate (Oh et al., 2014). These cells have been successfully used in this manner to clone a transgenic beagle (Oh et al., 2011). Given the wide use of these ASCs, exploring their biological characteristics and regulatory mechanisms is of great interest.

TSA and SAHA are the most commonly used HDACIs, representing a new generation of promising anticancer drugs, with roles in inhibition of cell proliferation, arrest of cell cycle progression, and induction of cell differentiation and apoptosis. In this study, we treated gADSCs with these compounds for 24,48 , and $72 \mathrm{~h}$, and found that cell proliferation gradually decreased as a result. The proportion of cells in early apoptosis and arrested in G0/ G1 increased after treatment with TSA or SAHA for $24 \mathrm{~h}$. As exposure time increased, early apoptotic cells gradually proceeded to late apoptosis. In addition, immunocytochemical and western blot analysis of gADSCs treated with TSA or SAHA for $24 \mathrm{~h}$ revealed increased $\mathrm{H} 3 \mathrm{~K} 9$ acetylation, with the former exerting a greater effect than the latter.

To further study $\mathrm{H} 3 \mathrm{~K} 9$ acetylation, we used qPCR and western blot to measure the extent of HDAC (HDAC1, HDAC6, and SIRT1) transcription and translation. HDAC1 is a class I HDAC that exhibits homology to the yeast enzyme reduced potassium dependency 3 (Rpd3), and is widely expressed in various tissues, inhibiting gene transcription in the nucleus. HDAC6 is a class II HDAC homologous to yeast Hdal that is specifically found in heart, muscle, and brain tissues and functions in cell differentiation. Owing to their homology with the yeast protein silent information regulator 2 (Sir2), class III HDACs are known as sirtuins. This subfamily has seven members (SIRT1-7). SIRT1 acts on histones, but also demonstrates non-HDAC activities (Yang et al., 2015). In this study, although TSA significantly promoted HDAC1, HDAC6, and SIRT1 transcription, it inhibited expression of SIRT1 protein, thus increasing H3K9 acetylation. The activity of SAHA differed from that of TSA, in that it increased HDACl and HDAC6 mRNA levels but inhibited SIRT1 transcription and HDAC1 and SIRT1 protein expression. Thus, TSA and SAHA raised the level of acetylated H3K9 in gADSCs and promoted transcription of SIRT1 and other deacetylase genes by inhibiting SIRT1 protein, relaxing the nucleosome structure.

NANOG, OCT4, and SOX2 are important transcription factors maintaining ESCs in a self-renewing state. The first of these is an essential factor in inhibiting the differentiation of pluripotent cells and restoring pluripotency of reprogrammed somatic cells. In differentiated cells, NANOG expression is low or absent. OCT4 is principally expressed in the inner cell mass of early embryos and is required for pluripotency in the early stages of embryonic development. However, its expression gradually weakens as differentiation progresses. SOX2 is a member of the SOX family of transcription factors, which are mainly expressed in the inner cell mass and

Genetics and Molecular Research 15 (4): gmr15048954 
ectoderm, they regulate the formation of pluripotent cells and promote embryonic development. As tissues differentiate, SOX2 expression gradually decreases. Inhibiting expression of SOX2 in mouse ESCs can promote their differentiation into trophoblasts. These findings suggest that SOX2 is important for maintaining the pluripotency of ESCs (Wang et al., 2012; Rizzino, 2013; Saunders et al., 2013; Zhang and Cui, 2014). Interactions between NANOG, OCT4, and SOX2 regulate their own expression and that of other multipotency-related transcription factors. Moreover, their expression is controlled by epigenetic modifications. For example, retinoic acid recruits the Sin3a/HDAC complex to directly inhibit transcription of NANOG and induce differentiation of mouse ESCs by deacetylating H3 (Lin et al., 2005), yet this same complex associates with SOX2 to activate NANOG expression (Baltus et al., 2009). In contrast, this study showed that histone $\mathrm{H} 3$ hyperacetylation caused by inhibiting histone deacetylase SIRT1 expression in gADSCs promoted NANOG expression. Therefore, our study demonstrated that in ESCs and ASCs alike expression of NANOG is regulated by epigenetic modification, a process that plays an important role in maintaining stem cell characteristics. In addition, some studies have reported that the use of RNA interference (RNAi) targeting SIRT1 in human bone marrow mesenchymal stem cells can decrease SOX2 protein levels, compromising the capacity of these cells for self-renewal and differentiation (Yoon et al., 2014). By western blotting, we found that decreasing SIRT1 expression downregulated SOX2, confirming the role of the former in the expression of the latter. Besides NANOG and SOX2, a previous study showed that downregulation of acetylation decreases OCT4 transcription (Zhu et al., 2015). The present investigation also demonstrated that increased H3K9 acetylation significantly promoted OCT4 transcription, but had no effect on OCT4 protein levels. Our results indicate that OCT4 transcription is regulated by both histone acetylation and other post-transcriptional regulatory mechanisms.

Early studies using an RNAi approach to target two important factors in histone synthesis revealed that slowing the DNA replication fork causes PCNA unloading. As PCNA is essential for DNA replication and acts as an accessory factor for DNA polymerase $\delta, \mathrm{S}$ phase progression is blocked, and DNA replication inhibited (Mejlvang et al., 2014; Makarova and Burgers, 2015). These observations indicate the importance of histones for DNA replication and assembly of nuclear proteins. In our study, TSA and SAHA dysregulated the balance between histone acetylation and deacetylation, increasing H3K9 acetylation, and inhibiting PCNA transcription and translation, resulting in direct inhibition of DNA replication and G0/G1 cell cycle arrest.

Telomerase is a eukaryotic ribonucleoprotein that was first discovered in and purified from Tetrahymena nuclear extracts. It is found in single-celled and multicellular organisms, and is responsible for the extension of telomeres and the promotion of cell growth and proliferation (Huang et al., 2014; Peterson et al., 2015). In general, the TERT promoter is located in a highly condensed region of the chromosome, interacting with hypoacetylated core histones (Wang et al., 2007). Thus, TERT transcription is thought to be regulated by epigenetic modifiers. In longterm cultures of fetal placental mesenchymal stem cells, histone $\mathrm{H} 3$ and $\mathrm{H} 4$ acetylation levels decrease and TERT transcription significantly declines (Zhu et al., 2015). We found that TSA and SAHA increased the level of acH3K9 in gADSCs, relaxed highly condensed chromatin, activated the TERT promoter, and stimulated TERT transcription. However, TERT expression was observed to be downregulated. Owing to cell cycle arrest in G0/G1 and cessation of DNA replication, E2F, which is responsible for transcription of genes involved in G1/S transition and DNA synthesis, negatively regulates TERT expression (Zhang et al., 2014).

Genetics and Molecular Research 15 (4): gmr15048954 
The tumor suppressor P53 is a multifunctional transcription factor. Environmental influences lead to HDAC1 acetylation and inhibition of SIRT1 expression; P53 is activated by acetylation, which in turn activates expression of downstream target genes, such as $P 21$ and $B A X$. Thus, P53 responds to external stimuli by inducing cell cycle arrest, apoptosis, and cell aging (Lin et al., 2005; Yang et al., 2015). For example, Adriamycin treatment of H9c2 cells downregulates heat shock protein 25 and weakens the interaction between SIRT1 and P53. This results in acetylation of the lysine residue at position 379 of P53, and activation of P53 transcription, thereby increasing BAX protein expression and inducing apoptosis (Zhang et al., 2016). Treatment of RKO cells, a human colon cancer line, with the HDACI CG200745 increases acetylation of the lysine at position 382 of P53, promotes P53-dependent gene transcription, enhances expression of the P53 target genes MDM2 and P21, and induces cell death (Oh et al., 2012). In contrast, our results showed that, although TSA and SAHA inhibited SIRT1 expression and elevated the level of $\mathrm{H} 3 \mathrm{~K} 9$ acetylation, they also lowered P53 transcription and translation, which were therefore affected by molecular mechanisms other than histone acetylation. P53 completes the endogenous apoptosis pathway by activating members of the BCL-2 family, such as the pro-apoptotic proteins BAX, BAK, BID, BIM, PUMA, NOXA, BAD, and BLK, and the anti-apoptotic factors BCL-2, BCL-XL, BCL-X, and BAG. Previous studies have revealed that HDACIs promote precursors of downstream apoptosis factors and decrease the presence of anti-apoptotic proteins (Ali et al., 2013; Fan et al., 2014). However, the downregulation of P53 observed in this study did not alter BAX protein expression. Our analysis demonstrated that TSA and SAHA induced P53/BAX-independent apoptosis.

\section{CONCLUSION}

TSA and SAHA inhibited the expression of SIRT1, thereby increasing H3K9 acetylation and triggering a series of biological events, including decreased cell viability, cell cycle arrest, and early apoptosis. In addition, H3K9 hyperacetylation affected NANOG, SOX2, TERT, PCNA, P53, and BAX expression, and the in vitro growth characteristics of ADSCs.

\section{Conflicts of interest}

The authors declare no conflict of interest.

\section{ACKNOWLEDGMENTS}

Research supported by a High Yield Transgenic Cashmere Goats Breeding grant (\#2014ZX08008-002).

\section{REFERENCES}

Ahmadi N, Razavi S, Kazemi M and Oryan S (2012). Stability of neural differentiation in human adipose derived stem cells by two induction protocols. Tissue Cell 44: 87-94. http://dx.doi.org/10.1016/j.tice.2011.11.006

Ali A, Bluteau O, Messaoudi K, Palazzo A, et al. (2013). Thrombocytopenia induced by the histone deacetylase inhibitor abexinostat involves p53-dependent and -independent mechanisms. Cell Death Dis. 4: e738. http://dx.doi. org/10.1038/cddis.2013.260

Baltus GA, Kowalski MP, Tutter AV and Kadam S (2009). A positive regulatory role for the mSin3A-HDAC complex in pluripotency through Nanog and Sox2. J. Biol. Chem. 284: 6998-7006. http://dx.doi.org/10.1074/jbc.M807670200

Genetics and Molecular Research 15 (4): gmr15048954 
Collas P (2010). Programming differentiation potential in mesenchymal stem cells. Epigenetics 5: 476-482. http://dx.doi. org/10.4161/epi.5.6.12517

Culmes M, Eckstein HH, Burgkart R, Nüssler AK, et al. (2013). Endothelial differentiation of adipose-derived mesenchymal stem cells is improved by epigenetic modifying drug BIX-01294. Eur. J. Cell Biol. 92: 70-79. http:// dx.doi.org/10.1016/j.ejcb.2012.11.001

Dudakovic A, Camilleri ET, Lewallen EA, McGee-Lawrence ME, et al. (2015). Histone deacetylase inhibition destabilizes the multi-potent state of uncommitted adipose-derived mesenchymal stromal cells. J. Cell. Physiol. 230: 52-62. http://dx.doi.org/10.1002/jep.24680

Fan QD, Wu G and Liu ZR (2014). Dynamics of posttranslational modifications of p53. Comput. Math. Methods Med. 2014: 245610. http://dx.doi.org/10.1155/2014/245610

Ge W, Liu Y, Chen T, Zhang X, et al. (2014). The epigenetic promotion of osteogenic differentiation of human adiposederived stem cells by the genetic and chemical blockade of histone demethylase LSD1. Biomaterials 35: 6015-6025. http://dx.doi.org/10.1016/j.biomaterials.2014.04.055

Huang Y, Liang P, Liu D, Huang J, et al. (2014). Telomere regulation in pluripotent stem cells. Protein Cell 5: 194-202. http://dx.doi.org/10.1007/s13238-014-0028-1

Kwon MJ, Kang SJ, Park YI, Yang YH, et al. (2015). Hepatic differentiation of human adipose tissue-derived mesenchymal stem cells and adverse effects of arsanilic acid and acetaminophen during in vitro hepatic developmental stage. Cell Biol. Toxicol. 31: 149-159. http://dx.doi.org/10.1007/s10565-015-9300-2

Lagutina I, Fulka H, Lazzari G and Galli C (2013). Interspecies somatic cell nuclear transfer: advancements and problems. Cell. Reprogram. 15: 374-384. http://dx.doi.org/10.1089/cell.2013.0036

Latella L, Palacios D, Forcales S and Puri PL (2012). Epigenetic control of reprogramming and cellular differentiation. Comp. Funct. Genomics 2012: 538639. http://dx.doi.org/10.1155/2012/538639

Lee K, Kim H, Kim JM, Kim JR, et al. (2011). Systemic transplantation of human adipose-derived stem cells stimulates bone repair by promoting osteoblast and osteoclast function. J. Cell. Mol. Med. 15: 2082-2094. http://dx.doi. org/10.1111/j.1582-4934.2010.01230.x

Leu S, Lin YC, Yuen CM, Yen CH, et al. (2010). Adipose-derived mesenchymal stem cells markedly attenuate brain infarct size and improve neurological function in rats. J. Transl. Med. 8: 63. http://dx.doi.org/10.1186/1479-5876-8-63

Lin T, Chao C, Saito S, Mazur SJ, et al. (2005). p53 induces differentiation of mouse embryonic stem cells by suppressing Nanog expression. Nat. Cell Biol. 7: 165-171. http://dx.doi.org/10.1038/ncb1211

Long CR, Westhusin ME and Golding MC (2014). Reshaping the transcriptional frontier: epigenetics and somatic cell nuclear transfer. Mol. Reprod. Dev. 81: 183-193. http://dx.doi.org/10.1002/mrd.22271

Makarova AV and Burgers PM (2015). Eukaryotic DNA polymerase $\zeta$. DNA Repair (Amst.) 29: 47-55. http://dx.doi. org/10.1016/j.dnarep.2015.02.012

Mejlvang J, Feng Y, Alabert C, Neelsen KJ, et al. (2014). New histone supply regulates replication fork speed and PCNA unloading. J. Cell Biol. 204: 29-43. http://dx.doi.org/10.1083/jcb.201305017

Ogura A, Inoue K and Wakayama T (2013). Recent advancements in cloning by somatic cell nuclear transfer. Philos. Trans. R. Soc. Lond. B Biol. Sci. 368: 20110329. http://dx.doi.org/10.1098/rstb.2011.0329

Oh ET, Park MT, Choi BH, Ro S, et al. (2012). Novel histone deacetylase inhibitor CG200745 induces clonogenic cell death by modulating acetylation of p53 in cancer cells. Invest. New Drugs 30: 435-442. http://dx.doi.org/10.1007/ $\underline{\text { s10637-010-9568-2 }}$

Oh HJ, Park JE, Kim MJ, Hong SG, et al. (2011). Recloned dogs derived from adipose stem cells of a transgenic cloned beagle. Theriogenology 75: 1221-1231. http://dx.doi.org/10.1016/j.theriogenology.2010.11.035

Oh HJ, Park JE, Park EJ, Kim MJ, et al. (2014). Analysis of cell growth and gene expression of porcine adipose tissuederived mesenchymal stem cells as nuclear donor cell. Dev. Growth Differ. 56: 595-604. http://dx.doi.org/10.1111/ dgd. 12159

Peterson DR, Mok HO and Au DW (2015). Modulation of telomerase activity in fish muscle by biological and environmental factors. Comp. Biochem. Physiol. C Toxicol. Pharmacol. 178: 51-59.

Ren Y, Wu H, Zhou X, Wen J, et al. (2012). Isolation, expansion, and differentiation of goat adipose-derived stem cells. Res. Vet. Sci. 93: 404-411.http://dx.doi.org/10.1016/j.rvsc.2011.08.014

Rinaldi L and Benitah SA (2015). Epigenetic regulation of adult stem cell function. FEBS J. 282: 1589-1604.http://dx.doi. org $/ 10.1111 /$ febs. 12946

Rizzino A (2013). Concise review: The Sox2-Oct4 connection: critical players in a much larger interdependent network integrated at multiple levels. Stem Cells 31: 1033-1039. http://dx.doi.org/10.1002/stem.1352

Rodriguez J, Boucher F, Lequeux C, Josset-Lamaugarny A, et al. (2015). Intradermal injection of human adipose-derived stem cells accelerates skin wound healing in nude mice. Stem Cell Res. Ther. 6: 241. http://dx.doi.org/10.1186/ $\underline{\text { s13287-015-0238-3 }}$

Genetics and Molecular Research 15 (4): gmr15048954 
Saunders A, Faiola F and Wang J (2013). Concise review: pursuing self-renewal and pluripotency with the stem cell factor Nanog. Stem Cells 31: 1227-1236. http://dx.doi.org/10.1002/stem.1384

Teven CM, Liu X, Hu N, Tang N, et al. (2011). Epigenetic regulation of mesenchymal stem cells: a focus on osteogenic and adipogenic differentiation. Stem Cells Int. 2011: 201371.http://dx.doi.org/10.4061/2011/201371

Wang S, Hu C and Zhu J (2007). Transcriptional silencing of a novel hTERT reporter locus during in vitro differentiation of mouse embryonic stem cells. Mol. Biol. Cell 18: 669-677. http://dx.doi.org/10.1091/mbc.E06-09-0840

Wang Z, Oron E, Nelson B, Razis S, et al. (2012). Distinct lineage specification roles for NANOG, OCT4, and SOX2 in human embryonic stem cells. Cell Stem Cell 10: 440-454. http://dx.doi.org/10.1016/j.stem.2012.02.016

Wankhade UD, Shen M, Kolhe R and Fulzele S (2016). Advances in adipose-derived stem cells isolation, characterization, and application in regenerative tissue engineering. Stem Cells Int. 2016: 3206807. http://dx.doi. org $/ 10.1155 / 2016 / 3206807$

Yang H, Yan B, Liao D, Huang S, et al. (2015). Acetylation of HDAC1 and degradation of SIRT1 form a positive feedback loop to regulate p53 acetylation during heat-shock stress. Cell Death Dis. 6: e1747. http://dx.doi.org/10.1038/ cddis.2015.106

Yannarelli G, Pacienza N, Cuniberti L, Medin J, et al. (2013). Brief report: The potential role of epigenetics on multipotent cell differentiation capacity of mesenchymal stromal cells. Stem Cells 31: 215-220. http://dx.doi.org/10.1002/ stem. 1262

Yoon DS, Choi Y, Jang Y, Lee M, et al. (2014). SIRT1 directly regulates SOX2 to maintain self-renewal and multipotency in bone marrow-derived mesenchymal stem cells. Stem Cells 32: 3219-3231.http://dx.doi.org/10.1002/stem.1811

Zhang C, Qu S, Wei X, Feng Y, et al. (2016). HSP25 down-regulation enhanced p53 acetylation by dissociation of SIRT1 from p53 in doxorubicin-induced H9c2 cell apoptosis. Cell Stress Chaperones 21: 251-260. http://dx.doi. org/10.1007/s12192-015-0655-3

Zhang Q, Ramlee MK, Brunmeir R, Villanueva CJ, et al. (2012). Dynamic and distinct histone modifications modulate the expression of key adipogenesis regulatory genes. Cell Cycle 11: 4310-4322.http://dx.doi.org/10.4161/cc.22224

Zhang S and Cui W (2014). Sox2, a key factor in the regulation of pluripotency and neural differentiation. World J. Stem Cells 6: 305-311.http://dx.doi.org/10.4252/wjsc.v6.i3.305

Zhang Y, Zhang A, Shen C, Zhang B, et al. (2014). E2F1 acts as a negative feedback regulator of c-Mycinduced hTERT transcription during tumorigenesis. Oncol. Rep. 32: 1273-1280.

Zhu Y, Song X, Han F, Li Y, et al. (2015). Alteration of histone acetylation pattern during long-term serum-free culture conditions of human fetal placental mesenchymal stem cells. PLoS One 10: e0117068. http://dx.doi.org/10.1371/ journal.pone. 0117068

Genetics and Molecular Research 15 (4): gmr15048954 